Departamento de Biología Celular, Histología y Farmacología. Facultad de Fisioterapia. Universidad de Valladolid. Campus de Soria. España.

2Departamento de Anatomía. Facultad de Fisioterapia. Universidad de Valladolid. Campus de Soria. España. ${ }^{3}$ Departamento de Fisiología. Facultad de Fisioterapia. Universidad de Valladolid. Campus de Soria. España. ${ }^{a} \mathrm{PhD}$.

Trabajo no recibió financiamiento. Loa autores declaran no tener conflictos de interés.

Recibido el 31 de diciembre de 2017, aceptado el 13 de noviembre de 2018.

Correspondencia a: Dr. Diego Fernández Lázaro Departamento de Biología Celular, Histología y Farmacología.

Facultad de Fisioterapia.

Universidad de Valladolid. 42003 Campus de Soria. España. diego.fernandez.lazaro@uva.es

\section{Agentes inmunomoduladores (IMiDs): herramientas para el tratamiento del mieloma múltiple}

\author{
DIEGO FERNÁNDEZ-LÁZARO ${ }^{1}$, CÉSAR IGNACIO FERNÁNDEZ-LÁZARO', \\ ALBERTO CABALLERO GARCIIA ${ }^{2}$, ALFREDO CÓRDOVA MARTÍNEZ ${ }^{3}$
}

\section{Immunomodulator drugs for the treatment of multiple myeloma}

Thalidomide changed the treatment of patients with multiple myeloma, however, its effectiveness has been compromised due to its side effects. New strategies are needed to specifically target the challenges of multiple myeloma through innovative, more effective, and less toxic therapy. The new immunomodulatory (IMiDs) compounds are structural and functional analogs of thalidomide, which were designed to improve the immunomodulatory and anticancer properties and tolerability profiles. We review the development of second generation IMiDs, lenalidomide and pomalidomide, their immunomodulatory and tumoricidal effects, their mechanisms of action, as well as the influence of dexamethasone on their effect and pharmacological resistance. In conclusion, lenalidomide and pomalidomide demonstrate a powerful activity and they are highly effective and well-tolerated treatment options for patients with myeloma, used alone or in combination with dexamethasone.

(Rev Med Chile 2018; 146: 1444-1451)

Key words: Dexamethasone; Multiple Myeloma; Neoplasm: Immunomodulation.
E 1 mieloma múltiple (MM) es una neoplasia de células B clonales caracterizada por la acumulación de células plasmáticas $(\mathrm{CP})$ malignas en la médula ósea (MO) y representa el $1 \%$ de todas las neoplasias y aproximadamente el $10 \%$ de las hemopatías malignas. La mediana de edad en el momento del diagnóstico es de 65 años y la supervivencia de los pacientes con MM es de unos 3 años aunque existen supervivencias superiores a 10 años ${ }^{1-6}$.

El tratamiento del MM está determinado fundamentalmente por la edad y por el estado general del paciente. El empleo de melfalán, bien aislado o combinado con prednisona, representó el primer avance importante en el tratamiento de esta enfermedad, aunque solo una minoría de los pacientes alcanzaba remisión completa (RC) (5\%), e inevitablemente, en todos los casos se produ- cían recaídas con una mediana de supervivencia aproximada de 2-3 años ${ }^{3,7-8}$. El empleo de combinaciones de fármacos como melfalán, vincristina, ciclofosfamida, prednisona, carmustina y adriamicina, seguido de altas dosis de quimioterapia (principalmente melfalán) con rescate de progenitores hematopoyéticos autólogos, ha ampliado las expectativas en el tratamiento del MM ya que se han conseguido remisiones completas en el 10 $-40 \%$ de los casos, con una supervivencia global (SG) de 54-58 meses ${ }^{3,8-14}$.

La aparición de nuevos fármacos con eficacia anti-mieloma ha supuesto un cambio radical en el tratamiento de esta enfermedad. El primero de todos ellos fue la talidomida que a finales de los 90 demostró capacidad de inducir respuestas en un tercio de los pacientes resistentes ${ }^{15-17}$. Posteriormente se descubrió que la inhibición del 
proteosoma, a través de un fármaco denominado bortezomib también era capaz de rescatar hasta $40 \%$ de enfermos refractarios incluso a talidomida y transplante, con una supervivivencia libre de progresión superior a 6 meses $^{18-19}$. El tercer fármaco aprobado para tratamiento de mieloma en recaída o refractarios ha sido la lenalidomida, que en combinación con dexametasona logra $60 \%$ de respuestas y una supervivencia libre de progresión cercana al año ${ }^{20-21}$.

Estos resultados propiciaron la investigación de estos fármacos también en enfermos de nuevo diagnóstico, tanto en candidatos a transplante como en pacientes mayores de 65-70 años, con resultado muy prometedores en ambos $\operatorname{casos}^{22-23}$.

\section{Inmunomoduladores (IMiDs) en mieloma múltiple}

\section{Talidomida}

La talidomida es un derivado del ácido glutámico que a principios de 1960 se utilizó en mujeres embarazadas para prevenir los vómitos, y que fue responsable de la focomelia. En el MM se empezó a utilizar basándose en ese potencial efecto antiangiogénico e inmunomodulador ${ }^{24}$. Además, regula la expresión de moléculas de adhesión, inhibe la producción de TNF- $\alpha$, estimula la proliferación de células T citotóxicas, favorece el bloqueo del ciclo celular en fase G1 e induce apoptosis ${ }^{25-27}$. El hecho de que la talidomida no es un fármaco citotóxico, y además tiene un potencial sinergismo in vitro con otros agentes (especialmente dexametasona) ha motivado el diseño de múltiples combinaciones.

\section{Análogos de la talidomida: inmunomoduladores (IMiDs)}

La búsqueda de análogos de la talidomida con aumento de la actividad inmunomoduladora y mejor perfil de seguridad llevó a probar añadir un grupo amino al cuarto carbono del anillo phthaloyl de la talidomida, obteniendo análogos 4-amino ${ }^{28}$. Estos fármacos conocidos como IMiDs (inmunomoduladores), poseen una potencia 50 a 200 veces superior a la de la talidomida para estimular los linfocitos T citotóxicos, y las células NK (naturales asesinas), y para disminuir la adhesión de las células plasmáticas al estroma, con la consiguiente reducción en la producción de citocinas e inducción de apoptosis ${ }^{16,29-30}$. Los resultados de un estudio multicéntrico fase II con uno de estos análogos (CC5013 o lenalidomida ${ }^{31}$, en el que se incluyeron, 100 pacientes con MM refractario o en recaída, que habían recibido un promedio de cuatro líneas de quimioterapia previa (incluyendo un trasplante en $40 \%$ de los casos y talidomida en $65 \%)$, mostró una tasa de respuestas globales del $37 \%$, (10\% RC, $12 \%$ RP y $15 \%$ mR). Además, en el $41 \%$ de los pacientes que no habían respondido a lenalidomida, la adición de dexametasona favoreció la obtención de respuestas.

La introducción de fármacos IMiDs que presentan un mecanismo de acción pleiotrópico, está en línea con el enfoque actual de la terapia contra el cáncer que persigue una triple acción: la inducción de apoptosis directa frente a células tumorales malignas, la interferencia con las interacciones del tumor con el micromedioambiente celular del estroma medular (BMSC), y el aumento de la respuesta inmune anti-tumoral ${ }^{32}$.

\section{Efectos tumoricidas de IMIDS y sobre la \\ interacción con el micromedioambiente}

El efecto tumoricidas de los IMiDs se produce a través de varios mecanismos, incluyendo la disminución de la producción de citoquinas y de factores de crecimiento que conducen a la interrupción de la ayuda del estroma, la inducción de genes supresores de tumores que conduzca a la parada del ciclo celular y la activación de las caspasas con inducción de apoptosis ${ }^{29,33-35}$.

Los IMiDs tienen un efecto directo sobre la proliferación de las células de MM a través de la regulación positiva de genes supresores tumora$\mathrm{les}^{35}$. En un estudio realizado por Gandhi et al. ${ }^{33}$, la lenalidomida inhibía el crecimiento celular tumoral por la inducción de ciclinas dependientes de kinasas (CDK), por la inhibición de p15, p16, p21 y p27, y por la respuesta temprana a los factores de transcripción EGR1, EGR2 y EGR3, en células derivadas de pacientes con $\mathrm{MM}^{33}$. Lenalidomida también indujo p15, p21, EGR1, EGR2 y EGR3 expresión en varias líneas de células MM.

Por otra parte, la lenalidomida en combinación con dexametasona, induce de forma sinérgica la sobreexpresión de estos genes supresores de tumores, lo que produce la inhibición de la fosforilación de la proteína retinoblastoma, la parada del ciclo celular G0/G1, y el inicio de apoptosis. Un estudio reciente ha demostrado que la mayor expresión de p21 que se produce por el tratamiento con 
lenalidomida y pomalidomida es debida a la reducción de metilación y la acetilación de histonas en el promotor de p21, lo que aumenta el acceso de factores de transcripción al $\mathrm{ADN}^{36-37}$.

Además, se ha demostrado que lenalidomida y pomalidomida activan las caspasas y sería un segundo mecanismo tumoricida. En el estudio realizado por Gandhi et $\mathrm{al}^{33}$. Lenalidomida activaba caspasas 3 y 8 en diferentes grados y en diferentes células MM líneas. Por otra parte, la lenalidomida más dexametasona produce la activación sinérgica de las caspasas 8 y 9 y la inducción final de la apoptosis ${ }^{38}$.

Por último, la interrupción de la interacción del estroma con la célula plasmática en el mieloma es, también, una importante estrategia en el tratamiento. Los IMiDs han demostrado que son capaces de disminuir la secreción de la interleukina 6 (IL-6) y del Vascular Endothelial Growth Factor (VEGF) inducida por las interacciones MM y BMSC ${ }^{29,38,39,40}$. Esta acción tendría efectos posteriores en la migración celular, el crecimiento y la supervivencia de la célula mielomatosa.

El mecanismo de acción más crucial de pomalidomida es la interrupción de las interacciones de la célula de MM con BMSC. Este mecanismo permite la inhibición de alguna citoquinas y factores de crecimiento necesarios para el crecimiento y proliferación de la célula mielomatosa ${ }^{41}$ Cuando comparamos pomalidomida con la talidomida, la inhibición in vitro del factor de necrosis tumoral alfa $(\mathrm{TNF} \alpha)$ fue mayor con la pomalidomida ${ }^{42}$. La importancia de esta inhibición es que TNF $\alpha$ es una de las citoquinas proinflamatorias que facilita el desarrollo del cáncer a través de la modulación de las interacciones de adhesión celular de las células de mieloma con el microambiente tumoral. Además, pomalidomida regula negativamente las moléculas de adhesión celular y reduce la secreción de IL-6 y el VEGF, ambos son factores de crecimiento importantes para la supervivencia y proliferación de las células $\mathrm{MM}^{43}$. Pomalidomida, también exhiben efectos antiangiogénicos mediante desregulación de factores quimiotácticos implicados en la migración de células endoteliales, como son nuevamente TNF $\alpha$, VEGF y además el factor de crecimiento de fibroblastos $\alpha$ $(\mathrm{FGF} \alpha)^{41,42}$. El efecto antiproliferativo de pomalidomida consiste en la inducción de la apoptosis y el bloqueo de moléculas de adhesión en células MM y BMSC ${ }^{43}$.

\section{Efectos inmunomoduladores de IMiDs}

Los efectos coestimuladores sobre las células $\mathrm{T}$ y las células asesinas naturales (NK) son otra propiedad importante de IMiDs para potenciar la actividad inmune anti-MM $\mathrm{MM}^{43}$.

Además de los efectos tumoricidas, lenalidomida ofrece la posibilidad de controlar la enfermedad de MM mediante la activación de la respuesta inmune. Un mecanismo por el que la lenalidomida tiene efectos inmunomoduladores es a través de un incremento de los linfocitos CD8 + lo que origina una respuesta antígeno-específica de células $\mathrm{T}^{44}$.

Otro importante mecanismo por el cual lenalidomida ejerce la inmunomodulación es por el aumento de las células $\mathrm{NK}^{45}$. Lenalidomida es capaz de estimular la actividad de NK a través de los receptores Fc- $\gamma$ (Fracción cristalizable) de señalización, que, a su vez, conduce a una mayor producción de granzima $\mathrm{B}$ y al aumento de la expresión de FAS-ligando en las células NK. Lenalidomida también ha demostrado tener efectos beneficiosos sobre las células $\mathrm{NK}^{46}$. Las células $\mathrm{NK}$ activadas por lenalidomida tienen una mayor capacidad de secretar interferón IFN $\gamma$, lo cual se asocia con la actividad antitumoral de las células NK.

La pomalidomida parece ser más potente que la talidomida y la lenalidomida con respecto a la coestimulación de células $\mathrm{T}^{42,43}$. Aunque el tratamiento con pomalidomida se ha traducido en un aumento significativa de la supervivencia en MM, el mecanismo de acción en lo que se refiere a la modulación inmune no se conoce por completo. En el futuro, el diseño de combinaciones terapéuticas óptimas con pomalidomida depende de una mayor comprensión de sus diversos mecanismos inmunomoduladores ${ }^{47}$.

\section{Diferencias en el modo de actuación de la Talidomida e IMDS}

Es de destacar que lenalidomida y pomalidomida tienen un mayor efecto tumoricida que talidomida. En un estudio in vitro en células MM1S, la síntesis de ADN se inhibió en 50\% con pomalidomida a concentración $0,01-0,1 \mathrm{mM}$ y con lenalidomida a 0,1-1,0 nM; Sin embargo, sólo 15\% se inhibió con talidomida a dosis de 100 mM 104. Lenalidomida también tiene un efecto más potente sobre el sistema inmune, específicamente por el aumento de la estimulación de la proliferación de células T y la producción de citoquinas ${ }^{48}$. Además, este fármaco es 100-1.000 veces más potente en 
la estimulación de la proliferación de células $\mathrm{T}$ e IFN- $\gamma$ y en la producción de IL-2 que talidomi$\mathrm{da}^{42,49}$. Lenalidomida y pomalidomida presentan perfiles de toxicidad más bajos que talidomida, exhibiendo menor somnolencia, estreñimiento, y neuropatía periférica, aunque la mielosupresión es más frecuente ${ }^{48}$.

\section{Influencia de dexametasona en el efecto de IMIDs}

La dexametasona ha sido tradicionalmente el fármaco más utilizado en el tratamiento del $\mathrm{MM}^{50}$, pero a menudo las células plasmáticas tumorales adquieren resistencia a esta terapia ${ }^{51}$. Lenalidomida en combinación con dexametasona inhibe el crecimiento de células de mieloma de forma sinérgica, a través de la activación de las caspasas 8 y 9, que promueven la inducción de apoptosis. Por tanto, dexametasona mejora los efectos citotóxicos directos de lenalidomida como agente único. Sin embargo, la dexametasona puede antagonizar los efectos inmunomoduladores del tratamiento con lenalidomida al inhibir IL-2, que es estimulador primario de producción de los linfocitos $\mathrm{T} y$, además lenalidomida inhibe IFN $\gamma$ que afecta a la producción de células NK. En este sentido, probablemente el uso de dosis bajas dexametasona proporcione efectos antiproliferativos sinérgicos sin antagonizar los efectos inmunes de lenalidomida, lo que, junto con la menor toxicidad inducida, puede explicar el beneficio de supervivencia observado ${ }^{29,33}$.

Pomalidomida ha demostrado beneficios clínicamente significativos en pacientes con mieloma múltiple recurrente y/o refractario (rrMM) cuando se combina con dexametasona, independientemente del estado refractario a lenalidomida o bortezomib y a otros nuevos fármacos para el tratamiento de $\mathrm{MM}^{52-53}$. La combinación de pomalidomida con dexametasona (PD) demuestra una potente actividad antiproliferativa y proapoptótica en líneas celulares MM resistentes a lenalidomida. PD también inhibió sinérgicamente el crecimiento tumoral en comparación con el tratamiento con agente único en xenoinjertos de células H929 R10-1 resistentes a la lenalidomida. Además, el perfil de expresión génica reveló un patrón único de expresión génica diferencial en muestras tratadas con PD, resaltado por la modulación de las vías proapoptóticas en células resistentes a lenalidomida ${ }^{54}$.
Mecanismos de resistencias para lenalidomida y pomalidomida en combinación con dexametasona

En la resistencia a fármacos (MDR) se han identificado diferentes mecanismos celulares que de forma aislada o combinada confieren resistencia tanto en células individuales como en poblaciones específicas ${ }^{55}$ y afectan a los pacientes con MM. Por lo tanto, el estudio de sus características y mecanismos es necesario para la búsqueda de nuevos enfoques terapéuticos para superarlas.

Surgen entonces, dos preguntas clínicamente relevantes para entender los mecanismos de resistencia: ¿ los diferentes agentes de la misma familia presentan resistencia cruzada? ¿esta resistencia es potencialmente reversible? La primera pregunta es especialmente importante porque nos encontramos en una era en la que se están desarrollando segundas o terceras generaciones de fármacos de la misma familia. Con respecto a la segunda pregunta, en la actualidad la reversibilidad es una cuestión clínica crítica ya que las opciones de tratamiento son actualmente limitadas, al desarrollar resistencias. Unas nuevas estrategias empleadas en la clínica moderna es que se aboga por el retratamiento con un fármaco previamente usado, asumiendo una posible re-sensibilización de las células tumorales ${ }^{56,57}$.

Para los IMiDs, el descubrimiento de Cereblon (CRBN) como la proteína de unión clave para lenalidomida y pomalidomida ha avanzado en la comprensión de los mecanismos de resistencia y la posibilidad encontrar formas de superarlo. CRBN es una proteína de 442 aminoácidos y es un componente del complejo CRL4 E3 ligasa que también contiene la proteína 1 de unión al daño del ADN (DDB1), regulador de cullins (Roc)-1 y Cullin $4(\mathrm{Cul} 4)^{58}$. Los estudios in vitro con lenalidomida han demostrado que la resistencia adquirida a este agente se asocia con una disminución en los niveles de proteína CRBN ${ }^{59-61}$. Además, la interacción de los IMiDs, lenalidomida y pomalidomida, a CRBN en células MM o T, se produjo la degradación de dos sustratos de la ligasa E3 de CRBN CRL4, concretamente Aiolos (codificada por IKZF3) e Ikaros (codificada por IKZF1), que dio como resultado la pérdida de viabilidad de las células MM y una producción mejorada de interleucina 2 en las células T, que explican tanto los efectos celulares autónomos como los inmunoestimuladores de $\mathrm{IMiDs}^{62-64}$.

Ocio et al. ${ }^{65}$ desarrollaron un modelo in vivo 
de resistencia a dos IMiDs (lenalidomida y pomalidomida) en combinación con dexametasona. Comprobaron que un descenso en los niveles de expresión de Aiolos e Ikaros cuando los tumores eran sensibles y un retorno a niveles basales cuando se volvieron resistentes a cualquiera los tratamientos con IMiDs y dexametasona. Aunque la degradación de Aiolos e Ikaros por la acción de lenalidomida y pomalidomida puede explicar la actividad de los IMiDs, este mecanismo sigue siendo objeto de investigación. Del mismo modo la recuperación de los niveles de Aiolos e Ikaros sugiere un posible mecanismo de resistencia. En este sentido, Thakurta et $\mathrm{al}^{66}$, observaron un posible mecanismo de adquisición resistencia a IMiDs por pérdida de CRBN. Por lo tanto, se podría considerar dos mecanismos de resistencia adicionales, no mutuamente excluyentes, que pueden ser la causa de resistencia adquirida a IMiDs. Esta teoría podría sustentarse en que los estudios ${ }^{65}$ que demuestran que las características de la resistencia generada no se observan resistencias cruzadas entre lenalidomida y pomalidomida en combinación con dexametasona, ya que ambos IMIDs son capaces de vencer la resistencia generada a la combinación alternativa. Lo que podría deberse a los requisitos diferenciales principalmente de Cereblon y sus sustratos Aiolos e Ikaros observados en células resistentes a cada combinación. Los perfiles diferenciales de expresión génica de LD y PD también podrían explicar la ausencia de resistencia cruzada ${ }^{65}$.

El inicio de la resistencia a ambas combinaciones de IMiDs con dexametasona, se asocia a una importante sobre-expresión de los diferentes componentes de esta vía MEK/ERK en las células resistentes. Esta vía podría ser un objetivo potencial de intervención por el cual la resistencia IMiD puede evitarse o superarse ${ }^{67}$. Una estrategia clínica permitiría utilizar los inhibidores de MEK para superar la resistencia a IMiDs ${ }^{65}$, por ejemplo, mediante la adición un inhibidor de MEK de molécula pequeña, podría resensibilizar células resistentes como es el caso de selumetinib ${ }^{65,68-69}$, u otro inhibidor empleado en MM, de esta vía de Kinasas $^{70-71}$.

Una observación importante es que la resistencia en modelo murino de resitencias ${ }^{65}$ es la reversibilidad de dichas resistencias frente al IMiD que ha generado la resistencia después de un período de lavado sin tratamiento; esto también puede ser por el resurgimiento de un clon sensible, que ocurriría al desconectar algunos de los mecanismos de resistencia inicialmente adquiridos, como lo es la vía MEK / ERK.

Estos resultados que proporcionan información sobre los mecanismos de resistencia adquirida a las combinaciones de LD y PD nos ofrecen posibles enfoques terapéuticos para abordar la resistencia a IMiDs en la clínica mediante: el tratamiento con IMiD de segunda generación (pomalidomida) en pacientes que han desarrollado resistencia a lenalidomida y también el retratamiento de pacientes refractarios después de un período de lavado durante el cual no están expuestos a ese determinado IMiD o a la combinación con inhibidores MEK para superar la resistencia generada a ese IMiD.

\section{Conclusión}

La introducción de IMiDs ha coincidido con el comienzo de un cambio importante en la comprensión de tratamientos efectivos contra el cáncer. La efectividad de IMiDs en MM radica en el conjunto de sus actividades: inmunomoduladoras, antiangiogénicas, antiinflamatorias, antiproliferativas, proapoptóticas, detención del ciclo celular e inhibición de migración celular y de la metástasis ${ }^{72}$.

Las resistencias afectan a los pacientes con MM tratados con IMiDs, sin embargo, los avances actuales en la compresión de los mecanismos de resistencia a IMiD permiten emplear diferentes alternativas clínicas para abordar la refractariedad con una elevada probabilidad de éxito, como podrían ser el uso de otro IMiD o mediante la combinación con fármacos específicos de la vía de resistencia adquirida. Por todo ello, los IMiDs demuestran una actividad potente y son opciones de tratamiento altamente eficaces, bien toleradas y con posibilidades de superar las resistencias adquiridas para pacientes con $\mathrm{MM}$, usados como agentes únicos o en combinación con dexametasona.

\section{Referencias}

1. Boccadoro M, Pileri A. Diagnosis, prognosis, and standard treatment of multiple myeloma. Hematol Oncol Clin North Am 1997; 11 (1): 111-31. 
2. Kyle RA, Rajkumar SV. Multiple myeloma. N Engl J Med 2004; 351 (18): 1860-73.

3. San Miguel JF, Blade J. En: Hoffbrand AV, Catovsky D, Editores. Multiple Myeloma Postgraduate Haematology. Sixth ed. London: Wiley-Blackwell; 2010. P. 578-98.

4. Sirohi B, Powles R. Multiple myeloma. Lancet 2004; 363 (9412): 875-87.

5. Blade J, Kyle RA. Multiple myeloma in young patients: clinical presentation and treatment approach. Leuk Lymphoma 1998; 30 (5-6): 493-01.

6. Bergsagel DE. The role of chemotherapy in the treatment of multiple myeloma. Baillieres Clin Haematol 1995; 8 (4): 783-94.

7. Alexanian R, Dimopoulos MA. Management of multiple myeloma. Semin Hematol 1995; 32: 20-30.

8. San Miguel JF, Blade Creixenti J, García-Sanz R. Treatment of multiple myeloma. Haematologica 1999; 84 (1): 36-58.

9. Attal M, Harousseau JL, Stoppa AM, Sotto JJ, Fuzibet JG, Rossi JF, et al. A prospective, randomized trial of autologous bone marrow transplantation and chemotherapy in multiple myeloma. Intergroupe Francais du Myelome. N Engl J Med 1996; 335 (2): 91-7.

10. Blade J, Rosinol L, Sureda A, Ribera JM, Díaz-Mediavilla J, García-Laraña J, et al. High-dose therapy intensification compared with continued standard chemotherapy in multiple myeloma patients responding to the initial chemotherapy: long-term results from a prospective randomized trial from the Spanish cooperative group PETHEMA. Blood 2005; 106 (12): 3755-59.

11. Boccadoro M, Marmont F, Tribalto M, Avvisati G, Andriani A, Barbui T, et al. Multiple myeloma: VMCP/ VBAP alternating combination chemotherapy is not superior to melphalan and prednisone even in high-risk patients. J Clin Oncol 1991; 9 (3): 444-8.

12. Child JA, Morgan GJ, Davies FE, Owen RG, Bell SE, Hawkins K, et al. High-dose chemotherapy with hematopoietic stem-cell rescue for multiple myeloma. N Engl J Med 2003; 348 (819): 1875-83.

13. Lenhoff S, Hjorth M, Holmberg E, Turesson I, Westin J, Nielsen JL, et al. Impact on survival of high-dose therapy with autologous stem cell support in patients younger than 60 years with newly diagnosed multiple myeloma: a population-based study. Nordic Myeloma Study Group. Blood 2000; 95 (81): 7-11.

14. Palumbo A, Bringhen S, Petrucci MT, Musto P, Rossini F, Nunzi M, et al. Intermediate-dose melphalan improves survival of myeloma patients aged 50 to 70 : results of a randomized controlled trial. Blood 2004; 104 (10): 3052-57.

15. Barlogie B, Desikan R, Eddlemon P, Spencer T, Zeldis
J, Munshi N, et al. Extended survival in advanced and refractory multiple myeloma after single-agent thalidomide: identification of prognostic factors in a phase 2 study of 169 patients. Blood 2001; 98 (2): 492-94.

16. Davies FE, Raje N, Hideshima T, Lentzsch S, Young G, Tai YT, et al. Thalidomide and immunomodulatory derivatives augment natural killer cell cytotoxicity in multiple myeloma. Blood 2001; 98 (1): 210-6.

17. Singhal S, Mehta J, Desikan R, Ayers D, Roberson P, Eddlemon $\mathrm{P}$, et al. Antitumor activity of thalidomide in refractory multiple myeloma. N Engl J Med 1999; 341 (21): 1565-71.

18. Hideshima T, Richardson P, Chauhan D, Palombella VJ, Elliott PJ, Adams J, et al. The proteasome inhibitor PS-341 inhibits growth, induces apoptosis, and overcomes drug resistance in human multiple myeloma cells. Cancer Res 2001; 61 (7): 3071-6.

19. Richardson PG, Barlogie B, Berenson J, Singhal S, Jagannath $\mathrm{S}$, Irwin D, et al. A phase 2 study of bortezomib in relapsed, refractory myeloma. N Engl J Med 2003; 348 (26): 2609-17.

20. Dimopoulos M, Spencer A, Attal M, Prince HM, Harousseau JL, Dmoszynska A, et al. Lenalidomide plus dexamethasone for relapsed or refractory multiple myeloma. N Engl J Med 2007; 357 (21): 2123-32.

21. Weber DM, Chen C, Niesvizky R, Wang M, Belch A, Stadtmauer EA, et al. Lenalidomide plus dexamethasone for relapsed multiple myeloma in North America. N Engl J Med 2007; 357 (21): 2133-42.

22. Facon T, Mary JY, Hulin C, Benboubker L, Attal M, Pegourie B, et al. Melphalan and prednisone plus thalidomide versus melphalan and prednisone alone or reduced-intensity autologous stem cell transplantation in elderly patients with multiple myeloma (IFM 99-06): a randomised trial. Lancet 2007; 370 (9594): 1209-18.

23. Garban F, Attal M, Michallet M, Hulin C, Bourhis JH, Yakoub-Agha I, et al. Prospective comparison of autologous stem cell transplantation followed by dose-reduced allograft (IFM99-03 trial) with tandem autologous stem cell transplantation (IFM99-04 trial) in high-risk de novo multiple myeloma. Blood 2006; 107 (9): 3474-80.

24. Rajkumar SV, Witzig TE. A review of angiogenesis and antiangiogenic therapy with thalidomide in multiple myeloma. Cancer Treat Rev 2000; 26 (5): 351-62.

25. D’Amato RJ, Lentzsch S, Anderson KC, Rogers MS. Mechanism of action of thalidomide and 3-aminothalidomide in multiple myeloma. Semin Oncol 2001; 28 (6): 597-601.

26. Peuckmann V, Fisch M, Bruera E. Potential novel uses of thalidomide: focus on palliative care. Drugs 2000; 60 (82): 273-92. 
27. Raje N, Anderson K. Thalidomide-a revival story. N Engl J Med 1999; 341 (21): 1606-9.

28. Davies F, Baz R. Lenalidomide mode of action: linking bench and clinical findings. Blood Rev 2010; 24 (Suppl 1): S13-9.

29. Hideshima T, Chauhan D, Shima Y, Raje N, Davies FE, Tai YT, et al. Thalidomide and its analogs overcome drug resistance of human multiple myeloma cells to conventional therapy. Blood 2000; 96 (9): 2943-50.

30. Mitsiades N, Mitsiades CS, Poulaki V, Chauhan D, Richardson PG, Hideshima T, et al. Apoptotic signaling induced by immunomodulatory thalidomide analogs in human multiple myeloma cells: therapeutic implications. Blood 2002; 99 (12): 4525-30.

31. Richardson PG, Schlossman RL, Weller E, Hideshima T, Mitsiades C, Davies F, et al. Immunomodulatory drug CC-5013 overcomes drug resistance and is well tolerated in patients with relapsed multiple myeloma. Blood 2002; 100 (9): 3063-7.

32. Raza S, Safyan RA, Lentzsch S. Immunomodulatory Drugs (IMiDs) in Multiple Myeloma. Curr Cancer Drug Targets 2017; 17 (9): 846-57.

33. Gandhi AK, Kang J, Capone L, Parton A, Wu L, Zhang $\mathrm{LH}$, et al. Dexamethasone synergizes with lenalidomide to inhibit multiple myeloma tumor growth, but reduces lenalidomide-induced immunomodulation of T and NK cell function. Curr Cancer Drug Targets 2010; 10 (2): 155-67.

34. Hideshima T, Nakamura N, Chauhan D, Anderson KC. Biologic sequelae of interleukin-6 induced PI3-K/Akt signaling in multiple myeloma. Oncogene 2001; 20 (42): 5991-00.

35. Verhelle D, Corral LG, Wong K, Wong K, Mueller JH, Moutouh-de Parseval L, et al. Lenalidomide and CC4047 inhibit the proliferation of malignant B cells while expanding normal CD34+ progenitor cells. Cancer Res 2007; 67 (2): 746-55.

36. Hideshima T, Chauhan D, Podar K, Schlossman RL, Richardson P, Anderson KC. Novel therapies targeting the myeloma cell and its bone marrow microenvironment. Semin Oncol 2001; 28 (6): 607-12.

37. Escoubet-Lozach L, Lin IL, Jensen-Pergakes K, Brady HA, Gandhi AK, Schafer PH, et al. Pomalidomide and lenalidomide induce p21 WAF-1 expression in both lymphoma and multiple myeloma through a LSD1-mediated epigenetic mechanism. Cancer Res 2009; 69 (18): 7347-56.

38. Das M. Lenalidomide plus dexamethasone in multiple myeloma. Lancet Oncol 2017; pii: S1470-2045 (17): 30894-X.

39. Gupta D, Treon SP, Shima Y, Hideshima T, Podar K, Tai
YT, et al. Adherence of multiple myeloma cells to bone marrow stromal cells upregulates vascular endothelial growth factor secretion: therapeutic applications. Leukemia 2001; 15 (12): 1950-61.

40. Andhavarapu S, Roy V. Immunomodulatory drugs in multiple myeloma. Expert Rev Hematol 2013; 6 (81): 69-82.

41. Clark SM, Steinbach A, Clemmons AB. Pomalidomide for the treatment of multiple myeloma. J Adv Pract Oncol 2014; 5 (1): 51-6.

42. Zhu YX, Kortuem KM, Stewart AK. Molecular mechanism of action of immune-modulatory drugs thalidomide, lenalidomide and pomalidomide in multiple myeloma. Leuk Lymphoma 2013; 54 (4): 683-7.

43. Dougé A, Lemal R, Chaleteix C. Pomalidomide for multiple myeloma. Bull Cancer 2017; 104 (9): 707-13.

44. Haslett PA, Hanekom WA, Muller G, Kaplan G. Thalidomide and a thalidomide analogue drug costimulate virus-specific CD8+ T cells “in vitro". J Infect Dis 2003; 187 (6): 946-55.

45. Wu L, Adams M, Carter T, Chen R, Muller G, Stirling $\mathrm{D}$, et al. Lenalidomide enhances natural killer cell and monocyte-mediated antibody-dependent cellular cytotoxicity of rituximab-treated CD20+ tumor cells. Clin Cancer Res 2008; 14 (14): 4650-7.

46. Chang DH, Liu N, Klimek V, Hassoun H, Mazumder A, Nimer SD, et al. Enhancement of ligand-dependent activation of human natural killer T cells by lenalidomide: therapeutic implications. Blood 2006; 108 82): 618-21.

47. Sun JJ, Zhang C, Zhou J, Yang HL. Pooled analysis of pomalidomide for treating patients with multiple myeloma. Asian Pac J Cancer Prev 2015; 16 (8): 3163-6.

48. Wang M, Dimopoulos MA, Chen C, Cibeira MT, Attal $\mathrm{M}$, Spencer A, et al. Lenalidomide plus dexamethasone is more effective than dexamethasone alone in patients with relapsed or refractory multiple myeloma regardless of prior thalidomide exposure. Blood 2008; 112 (12): 4445-51.

49. Kotla V, Goel S, Nischal S, Heuck C, Vivek K, Das B, et al. Mechanism of action of lenalidomide in hematological malignancies. J Hematol Oncol 2009; 2: 36.

50. Alexanian R, Dimopoulos MA, Delasalle K, Barlogie B. Primary dexamethasone treatment of multiple myeloma. Blood 1992; 80 (4): 887-90.

51. Alexanian R, Barlogie B, Dixon D. High-dose glucocorticoid treatment of resistant myeloma. Ann Intern Med 1986; 105 (1): 8-11.

52. San Miguel J, Weisel K, Moreau P, Lacy M, Song K, Delforge $\mathrm{M}$, et al. Pomalidomide plus low-dose dexamethasone versus high-dose dexamethasone alone for patients with relapsed and refractory multiple myeloma 
(MM-003): a randomised, open-label, phase 3 trial. Lancet Oncol 2013; 14 (11): 1055-66.

53. Lacy MQ, Hayman SR, Gertz MA, Dispenzieri A, Buadi F, Kumar S, et al. Pomalidomide (CC4047) plus low-dose dexamethasone as therapy for relapsed multiple myeloma. J Clin Oncol 2009; 27 (30): 5008-14.

54. Rychak E, Mendy D, Shi T, Ning Y, Leisten J, Lu L, et al. Pomalidomide in combination with dexamethasone results in synergistic anti-tumour responses in pre-clinical models of lenalidomide-resistant multiple myeloma. $\mathrm{Br}$ J Haematol 2016; 172 (6): 889-01.

55. Fodale V, Pierobon M, Liotta L, Petricoin E. Mechanism of cell adaptation: when and how do cancer cells develop chemoresistance? Cancer J 2011; 17 (2): 89-95.

56. Maiso P, Huynh D, Moschetta M, Sacco A, Aljawai Y, Mishima $Y$, et al. Metabolic signature identifies novel targets for drug resistance in Multiple Myeloma. Cancer research 2015; 75 (10): 2071-82.

57. Abraham J, Salama NN, Azab AK. (2015). The role of P-glycoprotein in drug resistance in multiple myeloma. Leuk Lymphoma 2015; 56 (1): 26-33.

58. Ito T, Ando H, Suzuki T, Ogura T, Hotta K, Imamura $\mathrm{Y}$ et al. Identification of a primary target of thalidomide teratogenicity. Science 2010; 327 (5971): 1345-50.

59. Zhu YX, Braggio E, Shi CX, Bruins LA, Schmidt JE, Van Wier $S$ et al. Cereblon expression is required for the antimyeloma activity of lenalidomide and pomalidomide. Blood 2011; 118 (18): 4771-79.

60. López-Girona A, Mendy D, Ito T, Miller K, Gandhi $\mathrm{AK}$, Kang J, et al. Cereblon is a direct protein target for immunomodulatory and antiproliferative activities of lenalidomide and pomalidomide. Leukemia 2012; 26 (11): 2326-35.

61. Lu G, Middleton RE, Sun H, Naniong M, Ott CJ, Mitsiades CS, et al. The myeloma drug lenalidomide promotes the cereblon-dependent destruction of Ikaros proteins. Science 2014; 343 (6168): 305-9.

62. Gandhi AK, Kang J, Havens CG, Conklin T, Ning Y, $\mathrm{Wu} \mathrm{L}$, et al. Immunomodulatory agents lenalidomide and pomalidomide co-stimulate $\mathrm{T}$ cells by inducing degradation of $\mathrm{T}$ cell repressors Ikaros and Aiolos via modulation of the E3 ubiquitin ligase complex CRL4(CRBN. Br J Haematol 2014; 164 (6): 811-21.

63. Kronke J, Udeshi ND, Narla A, Grauman P, Hurst SN, McConkey $\mathrm{M}$ et al. Lenalidomide causes selective degradation of IKZF1 and IKZF3 in multiple myeloma cells.
Science 2014; 343 (6168): 301-5.

64. Morgan B, Sun L, Avitahl N, Andrikopoulos K, Ikeda $\mathrm{T}$, Gonzales E, et al. Aiolos, a lymphoid restricted transcription factor that interacts with Ikaros to regulate lymphocyte differentiation. EMBO J 1997; 16 (8): 2004-13.

65. Ocio EM, Fernández-Lázaro D, San-Segundo L, López-Corral L, Bracket LA, Gutiérrez NC, et al. In vivo murine model of acquired resistance in myeloma reveals differential mechanisms for lenalidomide and pomalidomide in combination with dexamethasone. Leukemia 2015; 29 (3): 705-14.

66. Thakurta A, Gandhi AK, Waldman MF, Bjorklund C, Ning Y, Mendy D, et al. Absence of mutations in cereblon $(\mathrm{CRBN})$ and DNA damage-binding protein 1 (DDB1) genes and significance for IMiD therapy. Leukemia 2013; 28 (5): 1129-31.

67. Fernández-Lázaro D. Análisis pre-clínico comparativo de la eficacia, mecanismo de acción y mecanismos de resistencia de dos fármacos inmunomoduladores (IMIDS), lenalidomida y pomalidomida, en mieloma multiple. 2011. Tesis Doctoral. Universidad de Salamanca.

68. Holkova B, Zingone A, Kmieciak M, Bose P, Badros AZ, Voorhees PM, et al. A phase II trial of AZD6244 (Selumetinib, ARRY-142886), an oral MEK1/2 inhibitor, in relapsed/refractory multiple myeloma. Clinical Cancer Research 2016; 22 (5): 1067-75.

69. Suzuki R, Kikuchi S, Harada T, Mimura N, Minami J, Ohguchi $\mathrm{H}$, et al. Combination of a selective HSP90 $\alpha / \beta$ inhibitor and a RAS-RAF-MEK-ERK signaling pathway inhibitor triggers synergistic cytotoxicity in multiple myeloma cells. PloS one 2015; 10 (12): e0143847.

70. Kim K, Kong SY, Fulciniti M, Li X, Song W, Nahar S, et al. Blockade of the MEK/ERK signalling cascade by AS703026, a novel selective MEK1/2 inhibitor, induces pleiotropic anti-myeloma activity in vitro and in vivo. British journal of haematology 2010; 149 (4): 49-53.

71. Breitkreutz I, Podar K, Figueroa-Vázquez V, Wilhelm S, Hayden PJ, Anderson KC, et al. The orally available multikinase inhibitor regorafenib (BAY 73-4506) in multiple myeloma. Annals of hematology 2018; 97 (5): 839-49.

72. Chang X, Zhu Y, Shi C, Stewart AK. Mechanism of immunomodulatory drugs' action in the treatment of multiple myeloma. Acta Biochim Biophys Sin (Shanghai) 2014 ; 46 (3): 240-53. 